DOI: 10.24835/1607-0763-2017-2-53-59

Эластометрия печени с применением форсированного импульса акустической радиации (ARFI-эластометрия) у здоровых лиц в покое, после физической и пищевой нагрузок

Мегроян А.А. ${ }^{\text {, }, ~ К а м а л о в ~ Ю . Р ., ~ К р ы ж а н о в с к а я ~ Е . Ю ., ~ Ф и л и н ~ А . В . ~}$ ФГБНу “Российский научный центр хирургии им. акад. Б.В. Петровского”, Москва, Россия

\title{
Acoustic Radiation Force Impulse Elastometry (ARFI Elastometry) of the Liver in Normal Subjects, after Food Intake and Physical Effort
}

\author{
Megroyan A.A. ${ }^{\text {, }}$ Kamalov Ju.R., Krizhanovskaya E.Ju., Filin A.V.
}

B.V. Petrovsky National Research Centre of Surgery, Moscow, Russia

Цель исследования: определить показатели эластометрии с применением форсированного импульса акустической радиации (ARFI-эластометрия) в норме, после пищевой и физической нагрузок.

Материал и методы. ARFI-эластометрия печени была выполнена у 32 здоровых пациентов (20 (62,5\%) добровольцев и 12 (37,5\%) потенциальных доноров фрагмента печени). Средний возраст пациентов составил 26,9 \pm 5,3 года. Исследование выполняли строго натощак по межреберьям справа при задержке дыхания на фоне неглубокого вдоха в положении лежа на спине с отведенной правой рукой за голову. Эластометрию паренхимы печени 20 добровольцам также выполняли через 30 мин после пищевой нагрузки (калорийность которой составила 350 ккал) и на следующий день непосредственно после физической нагрузки (20 приседаний). Использовали диагностическую систему Siemens Acuson S2000 и широкополостный (1-6 МГц) конвексный датчик 6C1.

Результаты. Значение жесткости паренхимы правой доли печени в норме у 32 пациентов составило $1,16 \pm 0,12$ м/с (95\% ДИ 0,92 $\pm 1,38)$. У 20 пациентов значение жесткости печени натощак - 1,21 $\pm 0,09$ м/с (95\% ДИ 1,06 $\pm 1,38)$, через 30 мин после пищевой

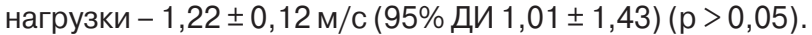
Физическая нагрузка не оказала достоверного влияния на жесткость печени $(1,16 \pm 0,12$ м/с против $1,20 \pm$ $\pm 0,07 \mathrm{M} / \mathrm{c}, \mathrm{p}>0,05)$.

Выводы. Жесткость правой доли печени в норме $1,16 \pm 0,12$ м/с (95\% ДИ 0,92 $\pm 1,38)$. Пищевая и физическая нагрузки не оказывают достоверного влияния на показатели жесткости печени.
Ключевые слова: ультразвуковая диагностика, ARFI-эластометрия, жесткость печени, пищевая нагрузка, физическая нагрузка.

Ссылка для цитирования: Мегроян А.А., Камалов Ю.Р., Крыжановская Е.Ю., Филин А.В. Эластометрия печени с применением форсированного импульса акустической радиации (ARFI-эластометрия) у здоровых лиц в покое, после физической и пищевой нагрузок. Медицинская визуализация. 2017; 21 (2): 53-59. DOI: 10.24835/1607-0763-2017-2-53-59.

$\star \star \star$

Purpose: the aim of this study was to assess the values of ARFI elastometry in normal subjects and effects of food intake and physical effort.

Material and methods. ARFI elastometry measurements were done in 32 patients (20 (62.5\%) volunteers and $12(37.5 \%)$ potential liver fragment donors). Mean age of patients was $26.9 \pm 5.3$ years. The study was performed in fasting condition in right intercostals approach, while the patients were asked to stop normal breathing for a moment lying in supine position with the right hand placed above the head. For 20 volunteers ARFI elastometry was performed at 30 minutes after food intake (about $350 \mathrm{kcal}$ ) and at next day immediately after physical effort (20 squats). The study was performed using Siemens Acuson S2000 ultrasound system with a 6C1 transducer.

Results. The stiffness of the right liver lobe in 32 healthy subjects was $1.16 \pm 0.12 \mathrm{~m} / \mathrm{s}(95 \% \mathrm{Cl} 0.92 \pm 1.38)$, the mean liver stiffness values of 20 volunteers in fasting condition were $1,21 \pm 0,09 \mathrm{~m} / \mathrm{s}(95 \% \mathrm{Cl} 1.06 \pm 1.38), 30$ minutes after the meal $-1.22 \pm 0.12 \mathrm{~m} / \mathrm{s}(95 \% \mathrm{Cl} 1.01 \pm 1.43)$ 
$(p>0.05)$. Physical effort didn't make significant influence on the liver stiffness measurements $(1.16 \pm 0.12 \mathrm{~m} / \mathrm{s}$ vs $1.20 \pm 0.07 \mathrm{~m} / \mathrm{s}, \mathrm{p}>0.05$ ).

Conclusion. Liver stiffness value in healthy subjects was $1.16 \pm 0.12 \mathrm{~m} / \mathrm{s}(95 \% \mathrm{Cl} 0.92 \pm 1.38)$. There was no significant influence of food intake and physical effort on the liver stiffness measurements.

Key words: ultrasound diagnostics, acoustic radiation force impulse elastometry (ARFI elastometry), liver stiffness, food intake, physical effort.

Recommended citation: Megroyan A.A., Kamalov Ju.R., Krizhanovskaya E.Ju., Filin A.V. Acoustic Radiation Force Impulse Elastometry (ARFI Elastometry) of the Liver in Normal Subjects, after Food Intake and Physical Effort. Medical visualization. 2017; 21 (2): 53-59

DOI: $10.24835 / 1607-0763-2017-2-53-59$

$\star * \star$

\section{Введение}

В ультразвуковой диагностике с 2003 г. используют метод транзиентной эластографии (ТЭ) [1], который позволяет получать дополнительную информацию о состоянии печени (определение степени фиброза) по сравнению со стандартным ультразвуковым исследованием (УЗИ). В последующие годы появились новые методики эластографии/эластометрии: компрессионная (квазистатическая, реально-временная) эластография (Hitachi, Япония), позволяющая качественно оценить жесткость объекта интереса; эластография с применением форсированного импульса акустической радиации (ARFI-эластометрия, point shear wave elastography) (Acuson-Siemens S 2000, S 3000 , США; iU 22, Philips, CША) и 2D-эластография сдвиговой волной (2D-SWE, SSI) (AixplorerTM, SuperSonic Imagine S.A., Aix-en-Provence, Франция). Последние 3 методики, в отличие от ТЭ, дают возможность проводить количественное измерение жесткости в области интереса, выбираемой в режиме В-сканирования, выражающийся в м/с или кПа. Все перечисленные эластографичесие методы, кроме ТЭ, интегрированы в обычные ультра- звуковые приборы, которые также могут быть использованы для выполнения стандартного УЗИ. Помимо ультразвуковых методик определения жесткости паренхимы печени существует еще один вид эластографии - это МР-эластография [2], котороя не нашла столь широкого применения в силу сложности своего проведения и высокой стоимости.

С 2003 г. в научной литературе опубликовано более 1300 статей по ТЭ [3]. Менее изучена методика ARFI-эластометрии, которая применяется в клинической практике с 2008 г. [4]. Технические и клинические аспекты применения эластографии/эластометрии подробно освещены в Европейских рекомендациях $[5,6]$.

При анализе данных литературы, посвященных данной методике, обращают на себя внимание большой разброс показателей скорости сдвиговой волны при выполнении ARFI-эластометрии в оценке жесткости печени у здоровых лиц [4, 7-23] и противоречия в определении влияния пищевой и физической нагрузок на эти показатели [24, 25]. Нам не удалось найти литературных данных, посвященным этим проблемам в отечественных источниках. В связи с этим для получения собственных результатов мы выполнили ARFIэластометрию у здоровых взрослых лиц, а также оценили влияние пищевой и физической нагрузок на жесткость паренхимы печени.

\section{Цель исследования}

Определить показатели эластометрии с применением форсированного импульса акустической радиации (ARFI-эластометрия) в норме, после пищевой и физической нагрузок.

\section{Материал и методы}

Для определения жесткости печени в норме были обследованы 32 человека (20 (62,5\%) добровольцев и 12 (37,5\%) потенциальных доноров

Для корреспонденции*: Мегроян Алина Алексановна - 119991 Москва, Абрикосовский пер., д. 2. Российский научный центр хирургии им. акад. Б. В. Петровского, лаборатория ультразвуковой диагностики. Тел.: 8-499-248-16-00; +7-926-320-29-84.

E-mail: alinamegroyan@gmail.com

Мегроян Алина Алексановна - врач ультразвуковой диагностики лаборатории ультразвуковой диагностики ФГБНу “Российский научный центр хирургии им. акад. Б.В. Петровского", Москва; Камалов Юлий Рафаэльевич - доктор мед. наук, заведующий лабораторией ультразвуковой диагностики ФГБНУ “Российский научный центр хирургии им. акад. Б.В. Петровского”, Москва; Крыжановская Евгения Юрьевна - канд. мед. наук, старший научный сотрудник лаборатории ультразвуковой диагностики ФГБНу "Российский научный центр хирургии им. акад. Б.В. Петровского", Москва; Филин Андрей Валерьевич - доктор мед. наук, заведующий отделением пересадки печени ФГБНу "Российский научный центр хирургии им. акад. Б.В. Петровского”, Москва.

Contact*: Alina A. Megroyan - 119991 Moscow, Abrikosovskii per., 2. B.V. Petrovsky National Research Centre of Surgery, Department of ultrasound diagnostics. Phone: +7-499-248-16-00; +7-926-320-29-84. E-mail: alinamegroyan@gmail.com

Alina A. Megroyan - doctor of ultrasound diagnostics of B.V. Petrovsky National Research Centre of Surgery, Moscow; Julius R. Kamalov - doct. of med. sci., head of ultrasound diagnostics of B.V. Petrovsky National Research Centre of Surgery, Moscow; Evgeniya U. Krizhanovskaya - cand. of med. sci., senior research assistant of ultrasound diagnostics of B.V. Petrovsky National Research Centre of Surgery, Moscow; Andrei V. Filin - doct. of med. sci., head of department of liver transplantation of B.V. Petrovsky National Research Centre of Surgery, Moscow. 
фрагмента печени), средний возраст составил 26,9 \ 5,3 года, среди них было 19 женщин и 13 мужчин. Индекс массы тела у женщин был равен

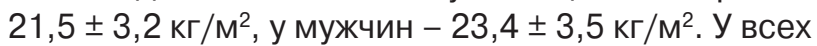
обследованных в анамнезе отсутствовали заболевания печени, поджелудочной железы, желчевыводящих путей; они имели нормальные показатели по данным общего и биохимического анализов крови.

Первым этапом всем 32 пациентам выполняли стандартное абдоминальное УЗИ (В-сканирование, дуплексное сканирование в режиме цветового допплеровского картирования и импульсной допплерографии) по стандартному протоколу на диагностической системе Acuson S2000 (Siemens, Германия) конвексным датчиком 6C1. Затем тем же датчиком производили эластометрию паренхимы печени по межреберьям справа при задержке дыхания на фоне неглубокого вдоха в положении пациента лежа на спине с отведенной правой рукой за голову. Производили оптимизацию В-изображения для того, чтобы область интереса располагалась вне изображения стенки сосудов и желчных протоков [26]. Измерения проводили в сегментах правой доли на глубине 2-3 см от капсулы печени [27] (рис. 1). Датчик располагали перпендикулярно поверхности тела, производили минимальное мануальное давление. Согласно C. Kaminuma и соавт. (2011) [28] и G. Ferraioli и соавт. (2015) [29], каждому пациенту проводили 5 измерений скорости сдвиговой волны, на основании которых автоматически (с помощью программы, заложенной в диагностической системе) рассчитывали среднее значение жесткости в м/с [30]. Исследование считали достоверным, если процент успешных измерений жесткости печени был более 60\% с интерквартильным соотношением менее $30 \%$ [31].

Вторым этапом 20 добровольцам по описанной выше методике выполняли эластометрию паренхимы печени после пищевой нагрузки. Все добровольцы получили одинаковый завтрак, калорийность которого составляла в среднем около 350 ккал. Эластометрия печени выполнялась через 30 мин после приема пищи.

Третьим этапом этим же 20 добровольцам на следующий день выполняли эластометрию паренхимы печени непосредственно после пробы с физической нагрузкой (20 приседаний), также у этих пациентов до и после нагрузки измеряли пульс и артериальное давление.

Полученные данные были собраны в файл Microsoft Excel. Показатели описательной статистики и графики были сделаны с помощью программы Statistica 10 (StatSoft ${ }^{\circledR}$, Польша). Высчи-

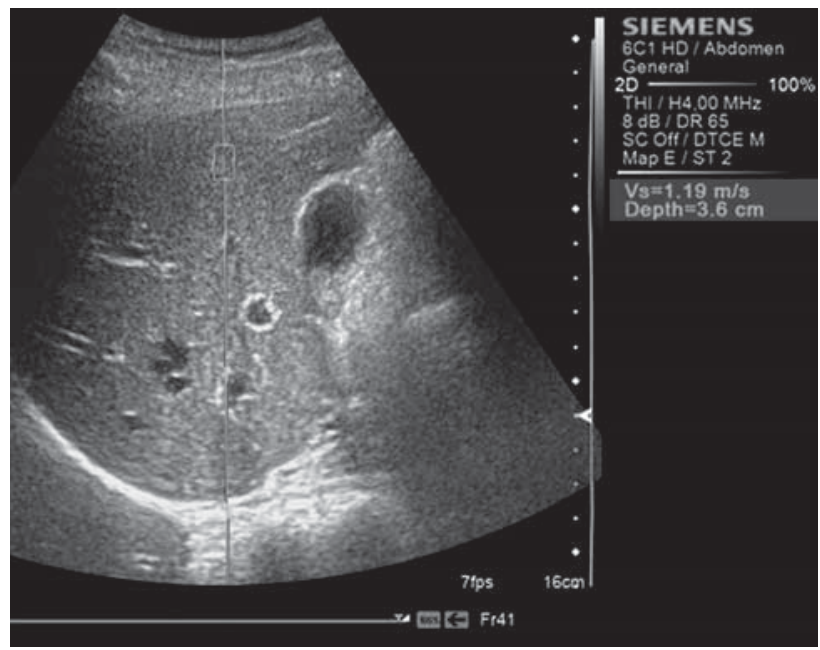

Рис. 1. ARFI-эластоизображение (у здоровых лиц): показатель жесткости печени - 1,19 м/с, область интереса расположена перпендикулярно поверхности тела на глубине 2 см от капсулы печени.

тывались среднее значение, стандартное отклонение $(\sigma)$, доверительный интервал (ДИ), достоверность (р). Разброс значений при измерении во всех случаях проведен с использованием интерквартильного размаха. Данные значения представлены в виде среднего значения в выборке 95\% ДИ. Достоверность различий между исследованиями до/после пищевой и физической нагрузок рассчитывалась с помощью t-критерия Стьюдента для сравнения парных выборок. Если значение рассчитанного критерия было меньше p $<0,05$, различия сравниваемых величин считались статистически значимыми.

Исследование было одобрено этическим комитетом ФГБНУ “РНЦХ им. акад. Б.В. Петровского”.

\section{Результаты}

При стандартном абдоминальном УЗИ у всех 32 пациентов выявлены нормальные размеры печени и селезенки, диаметры портальных, печеночных и нижней полой вен, однородность паренхимы печени и обычная ее интенсивность, а также отсутствие патологии желчевыводящей системы и поджелудочной железы.

Показатель жесткости паренхимы правой доли печени у 32 пациентов при ARFI-эластометрии

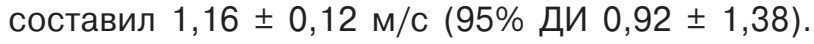
Значение ARFI-эластометрии у 20 добровольцев

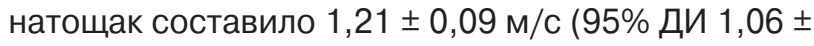
$\pm 1,38)$, через 30 мин после пищевой нагрузки значения жесткости печени у этих же пациентов

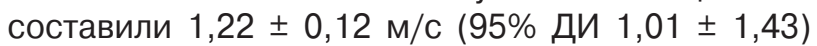
$p>0,05$, после физической нагрузки - 1,20 \pm 

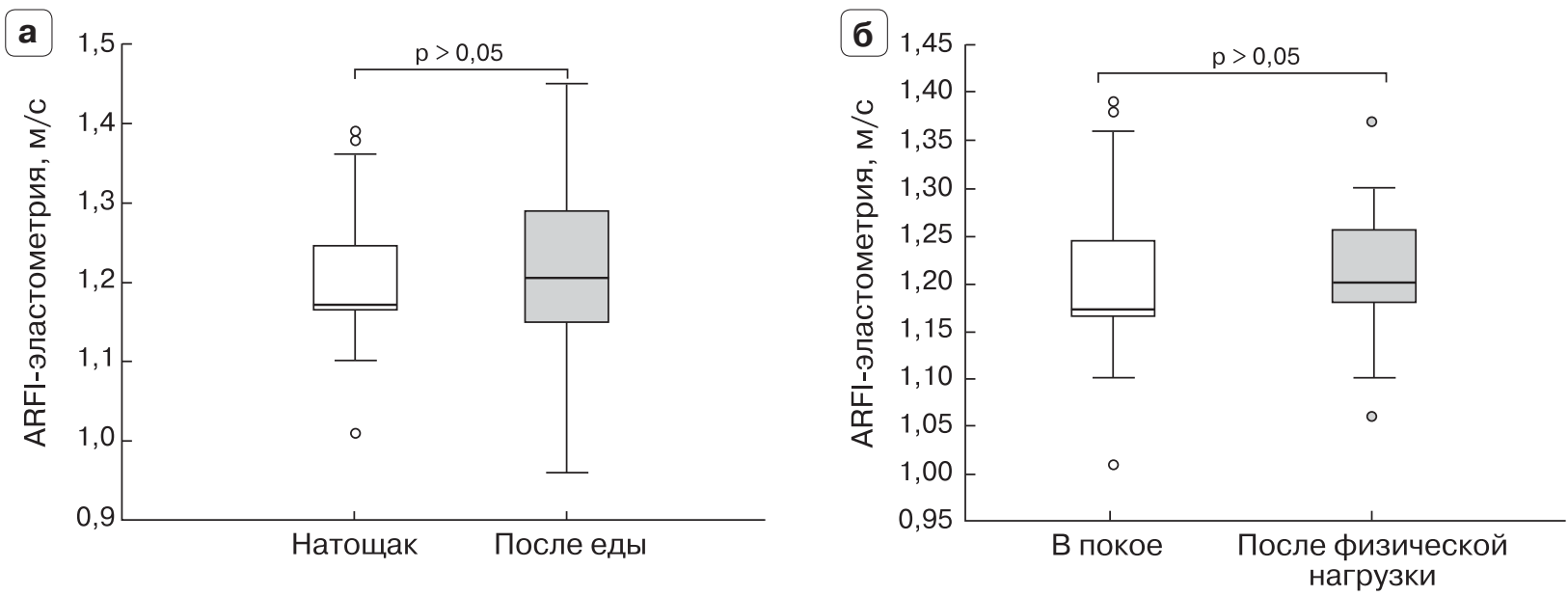

Рис. 2. Показатели ARFI эластометрии печени: а) натощак и после еды; б) в покое и после физической нагрузки. Коробчатые диаграммы или "ящики с усами" ("box-and-whisker plot") представляют собой межквартильный диапазон, в котором расположены 50\% значений. Границами ящиков служат 25-й и 75-й процентили, линия в середине ящика - среднее значение. Концы “усов" - края статистически значимой выборки (без выбросов). Маленькими кругами обозначены выбросы.

$\pm 0,07 \mathrm{~m} / \mathrm{c}(95 \%$ ДИ $1,08 \pm 1,34)$ р >0,05 (рис. 2). После физической нагрузки частота пульса у исследуемых пациентов увеличилась с $76,8 \pm$ $\pm 8,5$ до 97,3 $\pm 12,2$ уд/мин. Показатели артериального давления (систолическое/диастолическое) увеличились с $116,0 \pm 13,0 / 77,7 \pm 10,5$ до $123,1 \pm$ $\pm 14,1 / 79,5 \pm 11,1$ мм рт.ст.

Несмотря на то что достоверного различия после нагрузок не было выявлено, на графике отмечается больший разброс показателей после пищевой нагрузки, что также подтверждается увеличением коэффициента вариации до 9,8\% (натощак - 7\%), в то время как после физической нагрузки коэффициент вариации составил 5,8\% (до нагрузки - 7\%).

\section{Обсуждение}

В настоящее время при выполнении ARFIэластометрии печени не существует единого мнения относительно области исследования, глубины и места установки области интереса, а также необходимого количества измерений скорости сдвиговой волны. Учитывая мнение M. D'Onofrio и соавт. (2013) [23], мы производили исследование жесткости только правой доли печени, так как определение жесткости левой доли печени менее надежно. R.S. Goertz и соавт. (2012) [24], K. Rifai и соавт. (2011) [11] и Т. Toshima и соавт. (2011) [32] выявили, что показатели скорости сдвиговой волны в левой доле печени выше, чем в правой. По мнению этих авторов, это может быть обусловлено тем, что левая доля печени находится в непосредственной близости от диафрагмы, желудка, аорты и сердца [33]. Вследствие этого на показа- тели эластометрии могут оказывать влияние дыхание, присутствие пищи в желудке, пульсация аорты и помехи от сердца. Измерения ARFIэластометрии проводились в положении пациента лежа на спине, в связи с тем что в своем исследовании R.S. Goertz и соавт. (2012) [24] указывают на увеличение показателей скорости сдвиговой волны при исследовании пациентов стоя.

В своем исследовании мы ограничились пятью измерениями у каждого пациента в соответствии с рекомендациями С. Kaminuma и соавт. (2011) [28] и G. Ferraioli и соавт. (2015) [29], хотя A. Popescu и соавт. (2011) [12] и S. Horster и соавт. (2010) [34] советуют проводить 10 измерений. Полученные нами данные (жесткость правой доли печени составила 1,16 \pm 0,12 м/с) соответствуют большинству проведенных исследований, в которых скорость распространения сдвиговой волны была менее 1,2 м/с [4, 7-23]. Однако в сообщении A. Gallotti и соавт. (2010) [35] указывается более высокое значение жесткости печени в норме $1,59 \mathrm{~m} / \mathrm{c}$, а по данным исследования S. Colombo и соавт. (2012) [36] скорость сдвиговой волны составила 1,40 м/с. Е.В. Феоктистова и соавт. (2013) [37] сообщили о жесткости печени у детей в возрасте до 5 лет, которая составляла до 1,33 м/с с ее снижением в возрасте 10-16 лет до 1,17 м/с. По данным зарубежных исследователей, жесткость печени у здоровых детей независимо от возраста составляет 1,11-1,16 м/с [38-41].

В проведенном исследовании не было получено достоверных различий в скорости распространения сдвиговой волны у здоровых лиц до и после пищевой и физической нагрузок. Тем не менее 
в похожем исследовании А. Popescu и соавт. (2013) [25] отмечали увеличение показателей жесткости паренхимы печени после пищевой нагрузки, которые составили 1,51 $\pm 0,40$ м/с (натощак $-1,27 \pm 0,23 \mathrm{~m} / \mathrm{c})$. По данным исследования R.S. Goertz и соавт. (2012) [24] отмечено повышение показателей скорости сдвиговой волны после пищевой нагрузки до 1,12 $\pm 0,11 \mathrm{~m} / \mathrm{c}$ (натощак $1,03 \pm 0,10 \mathrm{~m} / \mathrm{c})$, которое, по мнению авторов, является следствием увеличения печеночного кровотока. При дуплексном сканировании сосудов печени через 15 мин после приема пищи M. Dauzat и соавт. (1994) [43] и C. Szinnai и соавт. (2001) [42] отмечали увеличение кровотока по воротной вене. Возможно, из-за того, что при использованной нами пищевой нагрузке (350 ккал) объемный афферентный печеночный кровоток не изменялся, нами не было отмечено значимых изменений параметров жесткости печени. Дуплексное сканирование сосудов печени мы не выполняли.

Однако результаты нашего исследования согласуются с мнением С. Kaminuma и соавт. (2011) [28], которые также не выявили влияния приема пищи на показатели жесткости печени.

В противоположность данным M. Gersak и coавт. (2016) [44] нами не выявлено достоверного влияния физической нагрузки на показатели жесткости печени. Наши расхождения с данными литературы по влиянию пищевой и физической нагрузок на жесткость печени, возможно, обусловлены недостаточной калорийностью пищевой нагрузки и интенсивностью физической нагрузки.

\section{Выводы}

1. Жесткость правой доли печени в норме составляет 1,16 $\pm 0,12 \mathrm{~m} / \mathrm{c}(4,0 \pm 0,04 \mathrm{k} П а)$. При соблюдении определенных правил выполнения ARFI-эластометрии эти значения могут использоваться в качестве референтных значений при обследовании пациентов с диффузными поражениями печени.

2. Для изучения влияния пищевой и физической нагрузок на показатели жесткости печени требуются дополнительные исследования, но для стандартизации выполнения ARFI-эластометрии печени рекомендуется избегать пищевых и физических нагрузок перед исследованием.

\section{Список литературы / References}

1. Sandrin L., Fourquet B., Hasquenoph J.M., Yon S., Fournier C., Mal F., Christidis C., Ziol M., Poulet B., Kazemi F., Beaugrand M., Palau R. Transient elastography: a new noninvasive method for assessment of hepatic fibrosis. Ultrasound Med. Biol. 2003; 29: 1705-1713.

2. Yin M., Talwalkar J.A., Glaser K.J., Manduca A., Grimm R.C., Rossman P.J., Fidler J.L., Ehman R.L. Assessment of hepatic fibrosis with magnetic resonance elastography. Clin. Gastroenterol. Hepatol. 2007; 5: 1207-1213. DOI: 10.1016/j.cgh.2007.06.012.

3. Castéra L., Foucher J., Bernard P.H., Carvalho F., Allaix D., Merrouche W., Couzigou P., Lédinghen V. Pitfalls of liver stiffness measurement: a 5-year prospective study of 13,369 examinations. Hepatology. 2010; 51: 828-835. DOI: 10.1002/hep.23425.

4. Friedrich-Rust M., Wunder K., Kriener S., Sotoudeh F., Richter S., Bojunga J., Herrmann E., Poynard T., Dietrich C.F., Vermehren J., Zeuzem S., Sarrazin C. Liver fibrosis in viral hepatitis: noninvasive assessment with acoustic radiation force impulse imaging versus transient elastography. Radiology. 2009; 252: 595-604. DOI: 10.1148/radiol.2523081928.

5. Bamber J., Cosgrove D., Dietrich C.F., Fromageau J., Bojunga J., Calliada F., Cantisani V., Correas J.-M., D'Onofrio M., Drakonaki E.E., Fink M., Friedrich-Rust M., Gilja O.H., Havre R.F., Jenssen C., Klauser A.S., Ohlinger R., Saftoiu A., Schaefer F., Sporea I., Piscaglia F. EFSUMB guidelines and recommendation clinical use of ultrasound elastography. Part 1: Basic principles and technology. Ultraschall Med. 2013; 34: 169-184. DOI: $10.1055 / \mathrm{s}-0033-1335205$.

6. Cosgrove D., Piscaglia F., Bamber J., Bojunga J., Correas J.-M., Gilja O.H., Klauser A.S., Sporea I., Calliada F., Cantisani F., D'Onofrio M., Drakonaki E.E, Fink M., Friedrich-Rust M., Fromageau J., Havre R.F., Jenssen C., Ohlinger R., Săftoiu A., Schaefer F., Dietrich C. F. EFSUMB guidelines and recommendation clinical use of ultrasound elastography. Part 2. Clinical applications. Ultraschall Med. 2013; 34: 238-253. DOI http://dx.doi.org/10.1055/s-0033-1335375.

7. Kuroda H., Kakisaka K., Tatemichi Y., Sawara K., Miyamoto Y., Oikawa K., Miyasaka A., Takikawa Y., Masuda T., Suzuki K. Non-invasive evaluation of liver fibrosis using acoustic radiation force impulse imaging in chronic hepatitis patients with hepatitis $\mathrm{C}$ virus infection. Hepatogastroenterology. 2010; 57: 1203-1207.

8. Popescu A., Sporea I., Sirli R., Bota S., Focşa M., Dănilă M., Nicoliţ̌ă D., Martie A., Sendroiu M., Juchiş A. The mean values of liver stiffness assessed by Acoustic Radiation Force Impulse elastography in normal subjects. Med. Ultrason. 2011; 13(1): 33-37.

9. Karlas T., Pfrepper C., Wiegand J., Wittekind C., Neuschulz M., Mössner J., Berg T., Tröltzsch M., Keim V. Acoustic radiation force impulse imaging (ARFI) for noninvasive detection of liver fibrosis: examination standards and evaluation of interlobe differences in healthy subjects and chronic liver disease. Scand. J. Gastroenterol. 2011; 46: 1458-1467. DOI: 10.3109/00365521.2011.610004.

10. Sporea I., Sirli R., Bota S., Fierbinţeanu-Braticevici C., Petrişor A., Badea R., Lupşor M., Popescu A., Dănilă M. Is ARFI elastography reliable for predicting fibrosis severity in chronic HCV hepatitis? WId J. Radiol. 2011; 3: 188-193. DOI: 10.4329/wjr.v3.i7.188.

11. Rifai K., Cornberg J., Mederacke I., Bahr M.J., Wedemeyer H., Malinski P., Bantel H., Boozari B., Potthoff A., Manns M.P., Gebel M. Clinical feasibility of liver elastography by acoustic radiation force impulse imaging (ARFI). Dig. Liver Dis. 2011; 43: 491-497. DOI: 10.1016/j.dld.2011.02.011.

12. Popescu A., Sporea I., Sirli R., Bota S., Focşa M., Dănilă M., Nicoliţă D., Martie A., Sendroiu M., Juchiş A. The mean 
values of liver stiffness assessed by Acoustic Radiation Force Impulse elastography in normal subjects. Med. Ultrason. 2011; 13(1): 33-37.

13. Sporea I., Bota S., Peck-Radosavljevic M., Sirli R., Tanaka H., lijima H., Badea R., Lupsor M., FierbinteanuBraticevici C., Petrisor A., Saito H., Ebinuma H., FriedrichRust M., Sarrazin C., Takahashi H., Ono N., Piscaglia F., Borghi A., D'Onofrio M., Gallotti A., Ferlitsch A., Popescu A., Danila M. Acoustic Radiation Force Impulse elastography for fibrosis evaluation in patients with chronic hepatitis $\mathrm{C}$ : an international multicenter study. Eur. J. Radiol. 2012; 81: 4112-4118.

DOI: 10.1016/j.ejrad.2012.08.018.

14. Eiler J., Kleinholdermann U., Albers D., Dahms J., Hermann F., Behrens C., Luedemann M., Klingmueller V., Alzen G.F. Standard value of ultrasound elastography using acoustic radiation force impulse imaging (ARFI) in healthy liver tissue of children and adolescents. Ultraschall Med. 2012; 33: 474-479. DOI: 10.1055/s-0032-1313145.

15. Sporea I., Sirli R., Bota S., Popescu A., Sendroiu M., Jurchis A. Comparative study concerning the value of acoustic radiation force impulse elastography (ARFI) in comparison with transient elastography (TE) for the assessment of liver fibrosis in patients with chronic hepatitis B and C. Ultrasound Med. Biol. 2012; 38: 13101316. DOI: 10.1016/j.ultrasmedbio.2012.03.011.

16. Karlas T., Hempel M., Tröltzsch M., Huster D., Günther P., Tenckhoff H., Mössner J., Berg T., Keim V., Wiegand J. Noninvasive evaluation of hepatic manifestation in Wilson disease with transient elastography, ARFI, and different fibrosis scores. Scand. J. Gastroenterol. 2012: 47: 1353-1361. DOI: 10.3109/00365521.2012.719924.

17. Jaffer O.S., Lung P.F., Bosanac D., Patel V.M., Ryan S.M., Heneghan M.A., Quaglia A., Sidhu P.S. Acoustic radiation force impulse quantification: repeatability of measurements in selected liver segments and influence of age, body mass index and liver capsule-to-box distance. Br. J. Radiol. 2012; 85: 858-863. DOI: 10.1259/bjr/74797353.

18. Marginean C.O., Marginean C. Elastographic assessment of liver fibrosis in children: A prospective single center experience. Eur. J. Radiol. 2012; 81: 870-874. DOI: 10.1016/j.ejrad.2012.04.014.

19. Crespo G., Fernández-Varo G., Mariño Z., Casals G., Miquel R., Martínez S.M., Gilabert R., Forns X., Jiménez W., Navasa M. ARFI, FibroScan, ELF, and their combinations in the assessment of liver fibrosis: a prospective study. J. Hepatol. 2012; 57: 281-287. DOI: 10.1016/j.jhep.2012.03.016.

20. Kircheis G., Sagir A., Vogt C., Vom Dahl S., Kubitz R., Häussinger D. Evaluation of acoustic radiation force impulse imaging for determination of liver stiffness using transient elastography as a reference. WId $\mathrm{J}$. Gastroenterol. 2012; 18: 1077-1084.

DOI: 10.3748/wjg.v18.i10.1077.

21. Yoon K.T., Lim S.M., Park J.Y., Kim do Y., Ahn S.H., Han K.H., Chon C.Y., Cho M., Lee J.W., Kim S.U. Liver stiffness measurement using acoustic radiation force impulse (ARFI) elastography and effect of necroinflammation. Dig. Dis. Sci. 2012; 57: 1682-1691. DOI: 10.1007/s10620-012-2044-4.

22. Son C.Y., Kim S.U., Han W.K., Choi G.H., Park H., Yang S.C., Han K.H. Normal liver elasticity values using acoustic radiation force impulse imaging: a prospective study in healthy living liver and kidney donors. J. Gastroenterol. Hepatol. 2012; 27: 130-136. DOI: $10.1111 / \mathrm{j} .1440-1746.2011 .06814 . x$

23. D'Onofrio M., Crosara S., De Robertis R., Canestrini S., Demozzi E., Gallotti A., Pozzi Mucelli R. Acoustic radiation force impulse of the liver. WId J. Gastroenterol. 2013; 19 (30): 4841-4849. DOI: 10.3748/wjg.v19.i30.4841.

24. Goertz R.S., Egger C., Neurath M.F., Strobel D. Impact of food intake, ultrasound transducer, breathing maneuvers and body position on acoustic radiation force impulse (ARFI) elastometry of the liver. Ultraschall Med. 2012; 33: 380-385. DOI: 10.1055/s-0032-1312816.

25. Popescu A., Bota S., Sporea I., Sirli R., Danila M., Racean S., Suseanu D., Gradinaru O., Ivascu Siegfried C. The influence of food intake on liver stiffness values assessed byacoustic radiation force impulse elastographypreliminary results. Ultrasound Med. Biol. 2013; 39 (4): 579-584. DOI: 10.1016/j.ultrasmedbio.2012.11.013.

26. Piscaglia F., Salvatore V., Di Donato R., D'Onofrio M., Gualandi S., Gallotti A., Peri E., Borghi A., Conti F., Fattovich G., Sagrini E., Cucchetti A., Andreone P., Bolondi L. Accuracy of Virtual Touch Acoustic Radiation Force Impulse (ARFI) imaging for the diagnosis of cirrhosis during liver ultrasonography. Ultraschall Med. 2011; 32 (2): 167-175. DOI: 10.1055/s-0029-1245948.

27. Takahashi H., Naofumi O., Yuichiro E., Mizuta T., Anzai K., Miyoshi A., Yoneda M., Nakajima A., Fujimoto K. Acoustic Radiation Force Impulse Elastography A Non-Invasive Alternative to Liver Biopsy. Liver Biopsy; Saga, Japan, InTech. 2011; 21: 335-342. DOI: 10.5772/20795.

28. Kaminuma C., Tsushima Y., Matsumoto N., Kurabayashi T., Taketomi-Takahashi A., Endo K. Reliable measurement procedure of virtual touch tissue quantification with acoustic radiation force impulse imaging. J. Ultrasound Med. 2011; 30 (6): 745-751.

29. Ferraioli G., Filice C., Castera L., Choi B.I., Sporea I., Wilson S.R., Cosgrove D., Dietrich C.F., Amy D., Bamber J.C., Barr R., Chou Y.H., Ding H., Farrokh A., Friedrich-Rust M., Hall T.J., Nakashima K., Nightingale K.R., Palmeri M.L., Schafer F., Shiina T., Suzuki S., Kudo $M$. WFUMB guidelines and recommendations for clinical use of ultrasound elastography: Part 3: Liver. Ultrasound. Med. Biol. 2015; 41(5): 1161-1179.

DOI: 10.1016/j.ultrasmedbio.2015.03.007.

30. Palmeri M.L., Wang M.H., Dahl J.J., Frinkley K.D., Nightingale K.R. Quantifying hepatic shear modulus in vivo using acoustic radiation force. Ultrasound Med. Biol. 2008; 34: 546-558. DOI: 10.1016/j.ultrasmedbio.2007.10.009.

31. Bota S., Sporea I., Sirli R., Popescu A., Dănilă M., Sendroiu M. Factors that influence the correlation of acoustic radiation force impulse (ARFI), elastography with liver fibrosis. Med. Ultrason. 2011; 13: 135-140.

32. Toshima T., Shirabe K., Takeishi K., Motomura T., Mano Y., Uchiyama H., Yoshizumi T., Soejima Y., Taketomi A., Maehara Y. New method for assessing liver fibrosis based on acoustic radiation force impulse: a special reference to the difference between right and left liver. J. Gastroenterol 2011; 46: 705-711. DOI: 10.1007/s00535-010-0365-7.

33. De Robertis R., D'Onofrio M., Demozzi E., Crosara S., Canestrini S., Pozzi Mucelli R. Noninvasive diagnosis of cirrhosis A review of different imaging modalities. WId J. Gastroenterol. 2014; 20 (23): 7231-7241. DOI: $10.3748 /$ wjg.v20.i23.7231.

34. Horster S., Mandel P., Zachoval R., Clevert D.A. Comparing acoustic radiation force impulse imaging to transient 
elastography to assess liver stiffness in healthy volunteers with and without valsalva manoeuvre. Clin. Hemorheol. Microcirc. 2010; 46 (2-3): 159-168.

DOI: $10.3233 / \mathrm{CH}-2010-1342$.

35. Gallotti A., D'Onofrio M., Mucelli Pozzi R. Acoustic radiation force impulse (ARFI) technique in ultrasound with Virtual Touch Tissue Quantification of upper abdomen. Radiol. Med. 2010; 115 (6): 889-897.

DOI: $10.1007 / \mathrm{s} 11547-010-0504-5$.

36. Colombo S., Buonocore M., Del Poggio A., Jamoletti C., Elia S., Mattiello M., Zabbialini D., Del Poggio P. Head-tohead comparison of transient elastography (TE), real-time ealastography (RTE) b acoustic force impulse (ARFI) imaging in the diagnosis of liver fibrosis. J. Gastroenterol. 2012; 47: 461-469. DOI: 10.1007/s00535-011-0509-4.

37. Феоктистова Е.В., Пыков М.И., Амосова А.А., Изотова О.Ю., Тарасов М.А., Дубровин М.М.. Применение ARFI-эластографии для оценки жесткости печени у детей различных возрастных групп. Ультразвуковая и функциональная диагностика. 2013; 6: 46-55.

Feoktistova E.V., Pykov M.I., Amosova A.A., Izotova O.Yu., Tarasov M.A., Dubrovin M.M. Application ARFI elastography to assess liver stiffness in children of different age groups. Ultrazvukovaya $i$ funktsionalnaya diagnostika. 2013; 6: 46-55. (in Russian)

38. Noruegas M.J., Matos H., Goncalves I., Cipriano M.A., Sanches $\mathrm{C}$. Acoustic radiation force impulse imaging in the assessment of liver fibrosis in children. Pediatr. Radiol. 2012; 42 (2): 201-204. DOI: 10.1007/s00247-011-2257-2.
39. Eiler J., Kleinholdermann U., Albers D., Dahms J., Hermann F., Behrens C., Luedemann M., Klingmueller V., Alzen G.F.P. Standard value of ultrasound elastography using acoustic radiation force impulse imaging (ARFI) in healthy liver tissue of children and adolescents. Ultraschall Med.2012;33(5):474-479. DOI: 10.1055/s-0032-1313145.

40. Hanquinet S., Courvoisier D., Kanavaki A., Dhouib A., Anooshiravani M. Acoustic radiation force impulse imaging - normal values of liver stiffness in healthy children. Pediatr. Radiol. 2013; 43 (5): 539-544. DOI: $10.1007 / \mathrm{s} 00247-012-2553-5$.

41. Lee M.J., Kim M.J., Han K.H., Yoon C.S. Age related changes in liver, kidney, and spleen stiffness in healthy children measured with acoustic radiation force impulse imaging. Eur. J. Radiol. 2013; 82 (6): 290-294. DOI:10.1016/j.ejrad.2013.01.018.

42. Szinnai C., Mottet C., Gutzwiller J.P., Drewe J., Beglinger C., Sieber C.C. Role of gender upon basal and postprandial systemic and splanchnic haemodynamics in humans. Scand. J. Gastroenterol. 2001; 36: 540-544.

43. Dauzat M., Lafortune M., Patriquin H., Pomier-Layrargues G. Meal induced changes in hepatic and splanchnic circulation: a noninvasive Doppler study in normal humans. Eur. J. Appl. Physiol. Occup. Physiol. 1994; 68: 373-380. DOI: $10.1007 /$ bf00843732.

44. Gersak M., Sorantin E., Windhaber J., Dudea S.M., Riccabona M. The influence of acute physical effort on the liver stiffness estimation using Virtual Touch Quantification (VTQ). Preliminary results. Med. Ultrason. 2016; 18 (2): 151-156. DOI: 10.11152/mu.2013.2066.182.vtq. 